The Journal of $\mathbf{N}_{\text {onlinear }} \mathbf{S}$ ciences and Applications http://www.tjnsa.com

\title{
BLOW-UP TIME OF SOME NONLINEAR WAVE EQUATIONS
}

\author{
THEODORE K. BONI ${ }^{1}$, DIABATE NABONGO ${ }^{2 *}$, AND ROGER B. SERY ${ }^{3 *}$
}

ABSTRACT. In this paper, we consider the following initial-boundary value problem

$$
\left\{\begin{array}{l}
u_{t t}(x, t)=\varepsilon L u(x, t)+b(t) f(u(x, t)) \text { in } \Omega \times(0, T), \\
u(x, t)=0 \text { on } \partial \Omega \times(0, T), \\
u(x, 0)=0 \text { in } \Omega \\
u_{t}(x, 0)=0 \text { in } \Omega,
\end{array}\right.
$$

where $\varepsilon$ is a positive parameter, $b \in C^{1}\left(\mathbb{R}_{+}\right), b(t)>0, b^{\prime}(t) \geq 0, t \in \mathbb{R}_{+}, f(s)$ is a positive, increasing and convex function for nonnegative values of $s$. Under some assumptions, we show that, if $\varepsilon$ is small enough, then the solution $u$ of the above problem blows up in a finite time, and its blow-up time tends to that of the solution of the following differential equation

$$
\left\{\begin{array}{l}
\alpha^{\prime}(t)=b(t) f(\alpha(t)), \quad t>0 \\
\alpha(0)=0, \quad \alpha^{\prime}(0)=0 .
\end{array}\right.
$$

Finally, we give some numerical results to illustrate our analysis.

\section{INTRODUCTION}

Let $\Omega$ be a bounded domain in $\mathbb{R}^{N}$ with smooth boundary $\partial \Omega$. Consider the following initial-boundary value problem

$$
\begin{gathered}
u_{t t}(x, t)=\varepsilon L u(x, t)+b(t) f(u(x, t)) \text { in } \Omega \times(0, T), \\
u(x, t)=0 \text { on } \partial \Omega \times(0, T), \\
u(x, 0)=0 \text { in } \Omega,
\end{gathered}
$$

Date: Received: May 2008; Accepted: 23 July 2008.

* Corresponding author.

2000 Mathematics Subject Classification. Primary 35B40, 35B50; Secondary 35K60.

Key words and phrases. Nonlinear wave equation, blow-up, convergence, numerical blow-up time. 


$$
u_{t}(x, 0)=0 \text { in } \Omega,
$$

where $\varepsilon$ is a positive parameter, $b \in C^{1}\left(\mathbb{R}_{+}\right), b(t)>0, b^{\prime}(t) \geq 0, t \in \mathbb{R}_{+}$. The operator $L$ is defined as follows

$$
L u=\sum_{i, j=1}^{N} \frac{\partial}{\partial x_{i}}\left(a_{i j}(x) \frac{\partial u}{\partial x_{j}}\right)
$$

where $a_{i j}: \bar{\Omega} \rightarrow \mathbb{R}, a_{i j} \in C^{1}(\bar{\Omega}), a_{i j}=a_{j i}, 1 \leq i, j \leq N$, and there exists a constant $C>0$ such that

$$
\sum_{i, j=1}^{N} a_{i j}(x) \xi_{i} \xi_{j} \geq C\|\xi\|^{2} \quad \forall x \in \bar{\Omega} \quad \forall \xi=\left(\xi_{1}, \ldots, \xi_{N}\right) \in \mathbb{R}^{N},
$$

where $\|\cdot\|$ stands for the Euclidean norm of $\mathbb{R}^{N}$. Here $(0, T)$ is the maximal time interval of existence of the solution $u$. The time $T$ may be finite or infinite. When $T$ is infinite, we say that the solution $u$ exists globally. When $T$ is finite, then the solution $u$ develops a singularity in a finite time, namely,

$$
\lim _{t \rightarrow T}\|u(\cdot, t)\|_{\infty}=\infty
$$

where $\|u(\cdot, t)\|_{\infty}=\sup _{x \in \Omega}|u(x, t)|$. In this last case, we say that the solution $u$ blows up in a finite time, and the time $T$ is called the blow-up time of the solution $u$.

Solutions of nonlinear wave equations which blow up in a finite time have been the subject of investigation of many authors (see [5], [7]-[9], [11]-[13], [17]-[19], and the references cited therein).

By standard methods, local existence, uniqueness, blow-up and global existence have been treated. In this paper, we are interested in the asymptotic behavior of the blow-up time when $\varepsilon$ is small enough. Our work was motivated by the paper of Friedman and Lacey in [6], where they have considered the following initial-boundary value problem

$$
\left\{\begin{array}{l}
u_{t}(x, t)=\varepsilon \Delta u(x, t)+f(u(x, t)) \text { in } \Omega \times(0, T), \\
u(x, t)=0 \text { on } \partial \Omega \times(0, T), \\
u(x, 0)=u_{0}(x) \text { in } \Omega,
\end{array}\right.
$$

where $\Delta$ is the Laplacian, $f:[0, \infty) \rightarrow(0, \infty)$ is a $C^{1}$ convex, increasing function, $\int_{0}^{\infty} \frac{d s}{f(s)}<\infty, u_{0}(x)$ is a continuous function in $\Omega$. Under some additional conditions on the initial data, they have shown that the solution of the above problem blows up in a finite time, and its blow-up time tends to that of the solution $\lambda(t)$ of the following differential equation

$$
\lambda^{\prime}(t)=f(\lambda(t)), \quad \lambda(0)=M,
$$

as $\varepsilon$ goes to zero, where $M=\sup _{x \in \Omega} u_{0}(x)$.

The proof developed in [6] is based on the construction of upper and lower solutions, and it is difficult to extend the method in [6] to the problem described in (1.1)-(1.4). In this paper, we prove similar results. More precisely, firstly, we show that when $\varepsilon$ is small enough, then the solution $u$ of (1.1)-(1.4) blows up 
in a finite time, and its blow-up time tends to that of the solution $\alpha(t)$ of the following differential equation

$$
\alpha^{\prime \prime}(t)=b(t) f(\alpha(t)), \quad \alpha(0)=0, \quad \alpha^{\prime}(0)=0 .
$$

A similar result has been obtained by N'gohisse and Boni in [15] in the case of the phenomenon of quenching (we say that a solution quenches in a finite time if it reaches a finite singular value in a finite time). Our paper is written in the following manner. In the next section, under some assumptions, we show that the solution $u$ of (1.1)-(1.4) blows up in a finite time, and its blow-up time goes to that of the solution $\alpha(t)$ of the differential equation defined in (1.6). Finally, in the last section, we give some numerical results to illustrate our analysis.

\section{BLOW-UP TIMES}

In this section, under some assumptions, we show that the solution $u$ of (1.1)(1.4) blows up in a finite time, and its blow-up time goes to that of the solution of the differential equation defined in (1.6) when $\varepsilon$ tends to zero. We also prove that the above result remains valid when $\varepsilon$ is fixed, and the domain $\Omega$ is large enough and is taken as parameter.

Before starting, let us recall a well known result. Consider the following eigenvalue problem

$$
\begin{gathered}
-L \varphi=\lambda \varphi \text { in } \Omega, \\
\varphi=0 \text { on } \partial \Omega, \\
\varphi>0 \text { in } \Omega .
\end{gathered}
$$

The above problem admits a solution $(\varphi, \lambda)$ with $\lambda>0$. We can normalize $\varphi$ so that $\int_{\Omega} \varphi d x=1$.

Our first result is the following.

Theorem 2.1. Let $A=\frac{\lambda}{b(0)} \int_{0}^{\infty} \frac{d s}{f(s)}$, and assume that the solution $\alpha(t)$ of the differential equation defined in (1.6) blows up at the time $T_{e}$. If $\varepsilon<A$, then the solution $u$ of (1.1)-(1.4) blows up in a finite time, and its blow-up time $T$ satisfies the following estimates

$$
0 \leq T-T_{e} \leq \frac{A T_{e}}{2}+o(\varepsilon)
$$

Proof. Since $(0, T)$ is the maximal time interval on which the solution $u$ exists, our aim is to show that $T$ is finite and satisfies the above inequalities. Introduce the function $v(t)$ defined as follows

$$
v(t)=\int_{\Omega} \varphi(x) u(x, t) d x \text { for } t \in[0, T) .
$$

Taking the derivative of $v$ in $t$, and using (1.1), we find that

$$
v^{\prime \prime}(t)=\varepsilon \int_{\Omega} \varphi(x) L u(x, t) d x+b(t) \int_{\Omega} f(u(x, t)) \varphi(x) d x \quad \text { for } \quad t \in(0, T) .
$$


According to Green's formula, and making use of (2.1), we note that

$$
\int_{\Omega} L u(x, t) \varphi(x) d x=\int_{\Omega} u(x, t) L \varphi(x) d x=-\lambda \int_{\Omega} \varphi(x) u(x, t) d x \quad \text { for } \quad t \in(0, T),
$$

which implies that

$$
v^{\prime \prime}(t)=-\lambda \varepsilon v(t)+b(t) \int_{\Omega} f(u(x, t)) \varphi(x) d x \quad \text { for } t \in(0, T) .
$$

Jensen's inequality renders

$$
v^{\prime \prime}(t) \geq-\lambda \varepsilon v(t)+b(t) f(v(t)) \text { for } t \in(0, T) .
$$

This estimate may be rewritten in the following manner

$$
v^{\prime \prime}(t) \geq b(t) f(v(t))\left(1-\frac{\lambda \varepsilon v(t)}{b(t) f(v(t))}\right) \quad \text { for } \quad t \in(0, T) .
$$

We observe that $b(t) \geq b(0)$ for $t \in(0, T)$, and $\int_{0}^{\infty} \frac{d \sigma}{f(\sigma)}=\sup _{t \geq 0} \int_{0}^{t} \frac{d \sigma}{f(\sigma)} \geq$ $\sup _{0 \leq t \leq \infty} \frac{t}{f(t)}$, because $f(s)$ is an increasing function for nonnegative values of $s$. Using these observations, we arrive at

$$
v^{\prime \prime}(t) \geq(1-\varepsilon A) b(t) f(v(t)) \text { for } \quad t \in(0, T) .
$$

Set

$$
w(t)=v\left(\frac{t}{\sqrt{1-\varepsilon A}}\right) \quad \text { for } \quad t \in[0, \sqrt{1-\varepsilon A} T) .
$$

A straightforward computation reveals that

$$
w^{\prime \prime}(t) \geq b\left(\frac{t}{\sqrt{1-\varepsilon A}}\right) f(w(t)) \quad \text { for } \quad t \in[0, \sqrt{1-\varepsilon A} T) .
$$

Since $b(s)$ is nondecreasing for nonnegative values of $s$, we discover that

$$
w^{\prime \prime}(t) \geq b(t) f(w(t)) \quad \text { for } \quad t \in[0, \sqrt{1-\varepsilon A} T) .
$$

It is not hard to check that

$$
w(0)=0 \text { for } w^{\prime}(0)=0 .
$$

Integrate the inequality (2.6) over $(0, t)$ to obtain

$$
w^{\prime}(t) \geq \int_{0}^{t} b(s) f(w(s)) d s \quad \text { for } \quad t \in[0, \sqrt{1-\varepsilon A} T) .
$$

Recall that $\alpha(t)$ is the solution of the following differential equation

$$
\begin{gathered}
\alpha^{\prime \prime}(t)=b(t) f(\alpha(t)), \quad t \in\left[0, T_{e}\right), \\
\alpha(0)=0, \quad \alpha^{\prime}(0)=0, \quad t \in\left[0, T_{e}\right),
\end{gathered}
$$

which implies that

$$
\alpha^{\prime}(t)=\int_{0}^{t} b(s) f(\alpha(s)) d s \quad \text { for } \quad t \in\left[0, T_{e}\right) .
$$


Since $w(0)=v(0)$, thanks to (2.8) and (2.9), an application of the maximum principle gives

$$
w(t) \geq \alpha(t) \text { for } t \in\left[0, T^{*}\right),
$$

where $T_{*}=\min \left\{T_{e}, \sqrt{1-\varepsilon A} T\right\}$. We deduce that

$$
T \leq \frac{T_{e}}{\sqrt{1-\varepsilon A} T}
$$

To prove this estimate, we argue by contradiction. Suppose that $T>\frac{T_{e}}{\sqrt{1-\varepsilon A T}}=$ $T^{\prime}$. From (2.10), we observe that $w$ blows up at the time $T_{e}$, because $w\left(T_{e}\right) \geq$ $\alpha\left(T_{e}\right)=\infty$, which implies that

$$
v\left(T^{\prime}\right)=v\left(\frac{T_{e}}{\sqrt{1-\varepsilon A} T}\right)=w\left(T_{e}\right)=\infty .
$$

Let us notice that $\|u(\cdot, t)\|_{\infty} \geq v(t)$ for $t \in(0, T)$. Since $v$ blows up at the time $T^{\prime}$, owing to the above estimate, it is easy to check that $u$ also blows up at the time $T^{\prime}$. But, this contradicts the fact that $(0, T)$ is the maximal time interval of existence of the solution $u$.

Now, introduce the function $U(t)$ defined as follows

$$
U(t)=\max _{x \in \Omega} u(x, t) \quad \text { for } \quad t \in[0, T) .
$$

We know that there exists $x_{0} \in \Omega$ such that $U(t)=u\left(x_{0}, t\right)$ for $t \in(0, T)$. It is not hard to see that $L u\left(x_{0}, t\right) \leq 0$ for $t \in(0, T)$. Making use of (1.1), we see that

$$
U^{\prime \prime}(t) \leq b(t) f(U(t)), \quad t \in(0, T) .
$$

Obviously, because of (1.3) and (1.4), we also have

$$
U(0)=0 \quad \text { and } \quad U^{\prime}(0)=0 .
$$

Integrating the inequality (2.14) from 0 to $t$, we find that

$$
U^{\prime}(t) \leq \int_{0}^{t} b(s) f(U(s)) d s \quad \text { for } \quad t \in(0, T) .
$$

Since $U(0)=\alpha(0)$, using (2.9) and (2.16), an application of the maximum principle renders

$$
U(t) \leq \alpha(t) \text { for } t \in\left(0, T_{0}\right),
$$

where $T_{0}=\min \left\{T, T_{e}\right\}$. We deduce that

$$
T \geq T_{e} .
$$

Indeed, assume that $T<T_{e}$. Taking into account (2.17), we observe that $U(T) \leq$ $\alpha(T)<\infty$. But, this contradicts the fact that $(0, T)$ is the maximal time interval of existence of the solution $u$. Apply Taylor's expansion to obtain

$$
\frac{1}{\sqrt{1-\varepsilon A}}=1+\frac{1}{2} \varepsilon A+o(\varepsilon) .
$$

Use (2.11), (2.18) and the above relation to complete the rest of the proof. 
Remark 2.2. If $b(t)=1$, then the solution $\alpha(t)$ defined in (1.6) satisfies

$$
\begin{gathered}
\alpha^{\prime \prime}(t)=f(\alpha(t)), \quad t \in\left(0, T_{e}\right), \\
\alpha(0)=0, \quad \alpha^{\prime}(0)=0 .
\end{gathered}
$$

Multiply both sides of $(2.20)$ by $\alpha^{\prime}(t)$ to obtain

$$
\left(\frac{\left(\alpha^{\prime}(t)\right)^{2}}{2}\right)^{\prime}=(F(\alpha(t)))_{t} \quad \text { for } \quad t \in\left(0, T_{e}\right)
$$

where $F(s)=\int_{0}^{s} f(\sigma) d \sigma$. Integrating the equality (2.22) over $(0, t)$, we find that

$$
\frac{\left(\alpha^{\prime}(t)\right)^{2}}{2}=F(\alpha(t)) \quad \text { for } \quad t \in\left(0, T_{e}\right)
$$

which implies that

$$
\alpha^{\prime}(t)=\sqrt{2 F(\alpha(t))} \text { for } t \in\left(0, T_{e}\right) .
$$

Let us notice that if the integral $\int_{0}^{\infty} \frac{d \sigma}{\sqrt{F(\sigma)}}$ is finite, then $\alpha(t)$ blows up at the time $T_{e}=\frac{1}{\sqrt{2}} \int_{0}^{\infty} \frac{d \sigma}{\sqrt{F(\sigma)}}$. In fact, we observe that

$$
\frac{d \sigma}{\sqrt{F(\sigma)}}=\sqrt{2} d t \quad \text { for } \quad t \in\left(0, T_{e}\right) .
$$

Integrate the above equality over $\left(0, T_{e}\right)$ to arrive at

$$
T_{e}=\frac{1}{\sqrt{2}} \int_{0}^{\infty} \frac{d \sigma}{\sqrt{F(\sigma)}}
$$

If $f(s)=e^{s}$, then $F(s)=e^{s}-1$. In this case $T_{e}=\frac{1}{\sqrt{2}} \int_{0}^{\infty} \frac{d \sigma}{\sqrt{e^{\sigma}-1}}$, and its value is slightly equal 2.22 .

Remark 2.3. Assume that Dirichlet boundary condition (1.2) is replaced by that of Robin, that is,

$$
\frac{\partial u}{\partial \eta}+\beta(x) u=0 \quad \text { for } \quad \partial \Omega \times(0, T)
$$

where $\beta \in C^{0}(\partial \Omega), \beta(x)>0$ on $\partial \Omega, \frac{\partial u}{\partial \eta}=\sum_{i, j=1}^{N} a_{i j} \cos \left(\nu, x_{i}\right) \frac{\partial u}{\partial x_{j}}$, $\nu$ is the exterior normal unit vector on $\partial \Omega$.

Consider the following eigenvalue problem

$$
\begin{gathered}
-L \psi=\lambda \psi \quad \text { in } \quad \Omega \\
\frac{\partial \psi}{\partial \eta}+\beta(x) \psi=0 \quad \text { for } \quad \partial \Omega, \\
\psi(x)>0 \quad \text { in } \quad \Omega .
\end{gathered}
$$


We know that the above eigenvalue problem admits a solution $(\psi, \lambda)$ with $\lambda>0$, and we can normalize $\psi$ so that $\int_{\Omega} \psi(x) d x=1$. Introduce the function $v(t)$ defined as follows

$$
v(t)=\int_{\Omega} u(x, t) \psi(x) d x \quad \text { in } \quad t \in[0, T) .
$$

Take twice the derivative of $v$ in $t$ and use (1.1), to obtain

$$
v^{\prime \prime}(t)=\varepsilon \int_{\Omega} L u(x, t) \psi(x) d x+b(t) \int_{\Omega} f(u(x, t)) \psi(x) d x \quad \text { for } \quad t \in(0, T) .
$$

By Green's formula, we have

$$
\int_{\Omega} L u(x, t) \psi(x) d x=\int_{\Omega} u(x, t) L \psi(x) d x+\int_{\partial \Omega} \psi(x) \frac{\partial u(x, t)}{\partial \eta} d s-\int_{\partial \Omega} u(x, t) \frac{\partial \psi(x)}{\partial \eta} d s .
$$

Using (2.24)-(2.26), we find that $\int_{\Omega} L u(x, t) \psi(x) d x=-\lambda \int_{\Omega} u(x, t) \psi(x) d x$.

Now, reasoning as in the proof of Theorem 2.1, we see that the result of Theorem 2.1 remains valid.

Now, let us show that we can obtain a result as the one given in Theorem 2.1 if the parameter $\varepsilon$ is fixed, and the domain $\Omega$ is large enough.

Assume that the domain $\Omega$ contains the ball $B(0, R)=\left\{x \in \mathbb{R}^{N} ;\|x\|<R\right\}$. Since $\Omega \supset B(0, R)$, we know that the eigenvalue $\lambda$ defined in (2.1) obeys the following estimates

$$
0<\lambda \leq \lambda_{R}=\frac{D}{R^{2}}
$$

where $D$ is a positive constant which depends only on the upper bound of the coefficients of the operator $L$ and the dimension $N$.

At the moment, we may state our result in the case where the domain $\Omega$ is large enough.

Theorem 2.4. Assume that $\operatorname{dist}(0, \partial \Omega)>0$. Suppose that the solution $\alpha(t)$ of the differential equation defined in (1.6) blows up at the time $T_{e}$ and let $E=$ $\frac{D}{b(0)} \int_{0}^{\infty} \frac{d \sigma}{f(\sigma)}$. If $\operatorname{dist}(0, \Omega)>\sqrt{\varepsilon E}$, then the solution $u$ of $(1.1)-(1.4)$ blows up in a finite time, and its blow-up time $T$ obeys the following estimates

$$
0 \leq T-T_{e} \leq \frac{\varepsilon E T_{e}}{2(\operatorname{dist}(0, \partial \Omega))^{2}}+o\left(\frac{1}{(\operatorname{dist}(0, \partial \Omega))^{2}}\right) .
$$

Proof. As in the proof of Theorem 2.1, it is not difficult to see that the solution $u$ of (1.1)-(1.4) blows up in a finite time $T$ which obeys the following estimates

$$
T_{e} \leq T \leq \frac{T_{e}}{\sqrt{1-\varepsilon A}}
$$

where $A=\frac{\lambda}{b(0)} \int_{0}^{\infty} \frac{d \sigma}{f(\sigma)}$.

Thanks to (2.28), $\lambda \leq \frac{D}{R^{2}}$, which implies that $A \leq \frac{D}{b(0) R^{2}} \int_{0}^{\infty} \frac{d \sigma}{f(\sigma)}=\frac{E}{R^{2}}$, where $R=\operatorname{dist}(0, \partial \Omega)$. We deduce from $(2.29)$ that

$$
T_{e} \leq T \leq \frac{T_{e}}{\sqrt{1-\frac{\varepsilon E}{R^{2}}}} .
$$


Apply Taylor's expansion to obtain

$$
\frac{1}{\sqrt{1-\frac{\varepsilon E}{R^{2}}}}=1+\frac{\varepsilon E}{2 R^{2}}+o\left(\frac{1}{R^{2}}\right)
$$

Use (2.30), and the above relation to complete the rest of the proof.

A direct consequence of Theorem 2.4 is that, if the solution $\alpha(t)$ of the differential equation defined in (1.6) blows up at the time $T_{e}$, and $\Omega=\mathbb{R}^{N}$, then the solution $u$ of (1.1) $-(1.4)$ blows up at the time $T$, and the following relation holds

$$
T=T_{e}
$$

\section{NumericAl RESUlts}

In this section, we give some computational results to confirm the theory established in the previous section. We consider the radial symmetric solution of (1.1)-(1.4) when $\Omega=B(0,1), L=\Delta, b(t)=1$, and $f(u)=e^{u}$. Hence, the problem (1.1)-(1.4) may be rewritten as follows

$$
\begin{gathered}
u_{t t}=\varepsilon\left(u_{r r}+\frac{N-1}{r} u_{r}\right)+e^{u}, \quad r \in(0,1), \quad t \in(0, T), \\
u_{r}(0, t)=0, \quad u(1, t)=0, \quad t \in(0, T), \\
u(r, 0)=0, \quad u_{t}(r, 0)=0, \quad r \in(0,1) .
\end{gathered}
$$

Let $I$ be a positive integer and let $h=1 / I$. Define the grid $x_{i}=i h, 0 \leq i \leq I$ and approximate the solution $u$ of $(3.1)-(3.3)$ by the solution $U_{h}^{(n)}=\left(U_{0}^{(n)}, \ldots, U_{I}^{(n)}\right)^{T}$ of the following explicit scheme

$$
\begin{gathered}
\frac{U_{0}^{(n+1)}-2 U_{0}^{(n)}+U_{0}^{(n-1)}}{\Delta t_{n}^{2}}=\varepsilon N \frac{2 U_{1}^{(n)}-2 U_{0}^{(n)}}{h^{2}}+e^{U_{0}^{(n)}} \\
\frac{U_{i}^{(n+1)}-2 U_{i}^{(n)}+U_{i}^{(n-1)}}{\Delta t_{n}^{2}}=\varepsilon\left(\frac{U_{i+1}^{(n)}-2 U_{i}^{(n)}+U_{i-1}^{(n)}}{h^{2}}+\frac{(N-1)}{i h} \frac{U_{i+1}^{(n)}-U_{i-1}^{(n)}}{2 h}\right) \\
+e^{U_{i}^{(n)}}, \quad 1 \leq i \leq I-1, \\
U_{I}^{(n)}=0, \\
U_{i}^{(0)}=0, \quad U_{i}^{(1)}=0, \quad 0 \leq i \leq I .
\end{gathered}
$$

In order to permit the discrete solution to reproduce the properties of the continuous one when the time $t$ approaches the blow-up time $T$, we need to adapt the size of the time step so that we take $\Delta t_{n}=\min \left\{h^{2}, e^{-\frac{1}{2}\left\|U_{h}^{(n)}\right\|_{\infty}}\right\}$ with $\left\|U_{h}^{(n)}\right\|_{\infty}=$ $\sup _{0 \leq i \leq I}\left|U_{i}^{(n)}\right|$. We also approximate the solution $u$ of (3.1)-(3.3) by the solution $U_{h}^{(n)}$ of the implicit scheme below

$$
\frac{U_{0}^{(n+1)}-2 U_{0}^{(n)}+U_{0}^{(n-1)}}{\Delta t_{n}^{2}}=\varepsilon N \frac{2 U_{1}^{(n+1)}-2 U_{0}^{(n+1)}}{h^{2}}+e^{U_{0}^{(n)}}
$$




$$
\begin{gathered}
\frac{U_{i}^{(n+1)}-2 U_{i}^{(n)}+U_{i}^{(n-1)}}{\Delta t_{n}^{2}}=\varepsilon\left(\frac{U_{i+1}^{(n+1)}-2 U_{i}^{(n+1)}+U_{i-1}^{(n+1)}}{h^{2}}+\frac{(N-1)}{i h} \frac{U_{i+1}^{(n+1)}-U_{i-1}^{(n+1)}}{2 h}\right) \\
+e^{U_{i}^{(n)}}, \quad 1 \leq i \leq I-1, \\
U_{I}^{(n+1)}=0, \\
U_{i}^{(0)}=0, \quad U_{i}^{(1)}=0, \quad 0 \leq i \leq I .
\end{gathered}
$$

As in the case of the explicit scheme, here, we also choose $\Delta t_{n}=\min \left\{h^{2}, e^{-\frac{1}{2}\left\|U_{h}^{(n)}\right\|_{\infty}}\right\}$. We need the following definition.

Definition 3.1. We say that the discrete solution $U_{h}^{(n)}$ of the explicit scheme or the implicit scheme blows up in a finite time if $\lim _{n \rightarrow \infty}\left\|U_{h}^{(n)}\right\|_{\infty}=\infty$, and the series $\sum_{n=0}^{\infty} \Delta t_{n}$ converges, where $\left\|U_{h}^{(n)}\right\|_{\infty}=\sup _{0 \leq i \leq I}\left|U_{i}^{(n)}\right|$. The quantity $\sum_{n=0}^{\infty} \Delta t_{n}$ is called the numerical blow-up time of the discrete solution $U_{h}^{(n)}$.

In the tables 1 and 2, in rows, we present the numerical blow-up times, the numbers of iterations $n$, the CPU times and the orders of the approximations corresponding to meshes of $16,32,64,128$. We take for the numerical blow-up time $T^{n}=\sum_{j=0}^{n-1} \Delta t_{j}$ which is computed at the first time when

$$
\Delta t_{n}=\left|T^{n+1}-T^{n}\right| \leq 10^{-16} .
$$

The order(s) of the method is computed from

$$
s=\frac{\log \left(\left(T_{4 h}-T_{2 h}\right) /\left(T_{2 h}-T_{h}\right)\right)}{\log (2)} .
$$

Numerical experiments for $\mathrm{N}=2 ; \varepsilon=\frac{1}{50}$

TABLE 1. Numerical blow-up times, numbers of iterations, CPU times (seconds) and orders of the approximations obtained with the explicit Euler method

\begin{tabular}{|c|c|c|c|c|}
\hline$I$ & $T^{n}$ & $n$ & $C P U_{t}$ & $s$ \\
\hline 16 & 2.220623 & 1143 & 3 & - \\
\hline 32 & 2.221240 & 4555 & 21 & - \\
\hline 64 & 2.221391 & 18203 & 169 & 2.03 \\
\hline 128 & 2.221428 & 72767 & 1183 & 2.03 \\
\hline
\end{tabular}


TABLE 2. Numerical blow-up times, numbers of iterations, CPU times (seconds) and orders of the approximations obtained with the implicit Euler method

\begin{tabular}{|c|c|c|c|c|}
\hline$I$ & $T^{n}$ & $n$ & $C P U_{t}$ & $s$ \\
\hline 16 & 2.220623 & 1143 & 3 & - \\
\hline 32 & 2.221240 & 4555 & 21 & - \\
\hline 64 & 2.221391 & 18203 & 169 & 2.03 \\
\hline 128 & 2.221428 & 72767 & 1183 & 2.03 \\
\hline
\end{tabular}

\section{REFERENCES}

1. L. M. Abia, J. C. López-Marcos and J. Martínez, On the blow-up time convergence of semidiscretizations of reaction-diffusion equations, Appl. Numer. Math., 26 (1998), 399414.

2. T. K. Boni, Extinction for discretizations of some semilinear parabolic equations, C.R.A.S, Serie I, 333 (2001), 795-800.

3. T. K. Boni, On blow-up and asymptotic behavior of solutions to a nonlinear parabolic equation of second order with nonlinear boundary conditions, Comment. Math. Univ. Comenian, 40 (1999), 457-475.

4. H. Brezis, T. Cazenave, Y. Martel and A. Ramiandrisoa, Blow-up for $u_{t}=u_{x x}+g(u)$ revisited, Adv. Diff. Eq., 1 (1996), 73-90.

5. K. Deng, Nonexistence of global solutions of a nonlinear hyperbolic system, Trans. Am. Math. Soc., 349 (1997), 1685-1696. 1

6. A. Friedman and A. A. Lacey, he blow-up time for solutions of nonlinear heat equations with small diffusion, SIAM J. Math. Anal., 18 (1987), 711-721. 1, 1

7. R. T. Glassey, Blow-up Theorems for nonlinear wave equations, Math. Z., 132 (1973), 183-203. 1

8. V. Georgiev and G. Todorova, Existence of a solution of the wave equation with nonlinear Dampiing and Source Trems, J. of Diff. Equat., 109 (1994), 295-308.

9. G. Guowang and W. Shubin, Existence and nonexistence of global solutions for the generalized IMBq equation, Nonl. Anal. TMA, 36 (1999), 961-980. 1

10. S. Kaplan, On the growth of solutions of quasi-linear parabolic equations, Comm. Pure Appl. Math., 16 (1963), 305-330.

11. H. A. Levine, Instability and nonexistence of global solutions to nonlinear wave equations of the form $\rho u_{t t}=-A v+F(u)$, Trans. Am. Math. Soc., 192 (1974), 1-21. 1

12. H. A. Levine, Some additional remarks on the nonexistence of global solutions to nonlinear wave equations, SIAM J. Math. Anal., 5 (1974), 138-146.

13. R. C. Maccamy and V. J. Mizel, Existence and nonexistence in the lare of solutions of quasilinear wave equations, Arch. Rational Meth. Anal., 25 (1967), 299-320. 1

14. T. Nakagawa, Blowing up on the finite difference solution to $u_{t}=u_{x x}+u^{2}$, Appl. Math. Optim., 2 (1976), 337-350.

15. F. K. N'gohisse and T. K. Boni, Quenching time of some nonlinear wave equations, To appear. 1.

16. M. H. Protter and H. F. Weinberger, Maximum principles in differential equations, Prentice Hall, Englewood Cliffs, NJ, (1967).

17. M. Reed, Abstract nonlinear wave equations, Lecture notes in Mathematics 507, SpringerVerlag Berlin, New-York, (1976). 1 
18. D. H. Sattinger, On global solution of nonlinear hyperbolic equations, Arch. Rational Mech. Anal., 30 (1968), 148-172.

19. A. Sang and R. Park, Remarks on quasilinear hyperbolic equations with dynamic boundary conditions, Math. Narchr., 198 (1999), 169-178. 1

20. W. Walter, Differential-und Integral-Ungleichungen, Springer, Berlin, (1954).

${ }^{1}$ Institut National Polytechnique Houphouet-Boigny de Yamoussoukro, BP 1093 Yamoussoukro, (Cote D'Ivolre).

E-mail address: theokboni@yahoo.fr.

2 Universite D'Abobo-Adjame, UFR-SFA, Departement de Mathematiques et Informatiques, 16 BP 372 Abidjan 16, (Cote D'IVOIRE)

E-mail address: nabongo_diabate@yahoo.fr

${ }^{3}$ Institut National Polytechnique Houphouet-Boigny de Yamoussoukro, BP 1093 Yamoussoukro, (Cote D'IvoIre).

E-mail address: serybrci@yahoo.fr 